\title{
Innovative Self Management System for Guided Cardiac Rehabilitation
}

\author{
M. Ottaviano, C. Vera-Muñoz, M.T. Arredondo, D. Salvi, S. Salvi, J.M. Páez, A. Díaz de Barrionuevo
}

\begin{abstract}
This paper describes the design and development of a system for cardio rehabilitation of patients that suffered a myocardial infarction. The proposed solution focuses on exercise prescriptions and the encouragement of healthy behaviors. The innovative strategy of the design takes into account health promotion models to provide safe, assistive exercise training sessions, personalized feedbacks, and educational contents.
\end{abstract}

\section{INTRODUCTION}

$\mathrm{T}$ HE healthcare industry is increasingly consumerdriven. The 1st Word population is ageing and the previsions indicate that costs in health care will progressively grow [1]. Experts in the area propose new paradigms for a new sustainable approach of care that focus on the improvement of quality of services and cost reductions. In these new models two aspects are important: the use of technology and the promotion of self management for care delivery [2]. Personalized Health Systems (PHS) are the novel solutions that promise changes in the future heath care systems, posing the challenge on how the use of technology can actually facilitate patients' engagement and empowerment. In many cases the lack of commitment is caused by insufficient information or a wrong approach in its delivery [3].

Health promotion models consider education as a fundamental "cue to action" that can improve the perceived threat or condition and promote behavioral changes. However, nowadays, even if technologies facilitate the "health information era", there are still barriers that jeopardize the citizens' perception of usefulness of the available information (e.g. social factors, understanding of the information, and difficulty of use). The real key of education and information delivery is not the technological data flow, but how this data is shown to the user.

This paper presents the design and development of a cardiac rehabilitation system for post myocardial infarction (M.I) patients (Guided Exercise). The system has been

Manuscript received April 15, 2011. This work is partially funded by the European Commission through the HeartCycle Project, "Compliance and effectiveness in HF and CHD closed-loop management", FP7 - 216695.

M. Ottaviano, C. Vera-Muñoz, M.T. Arredondo, D. Salvi, S. Salvi are with Life Supporting Technologies, Universidad Politécnica de Madrid (UPM). Madrid 28040. Spain (e-mail: mottaviano@1st.tfo.upm.es, cvera@1st.tfo.upm.es, mta@1st.tfo.upm.es, dsalvi@1st.tfo.upm.es, ssalvi@1st.tfo.upm.es). J.M. Páez is with ETSI Telecomunicación. Universidad Politécnica de Madrid. 28040. Spain (e-mail: jm.paez@upm.es). A. Díaz de Barrionuevo is with ETSI Montes. Universidad Politécnica de Madrid. 28040. Spain (e-mail: a.dbarrionuevo@upm.es) developed in the context of the European funded project HeartCycle [4], and focuses on guiding patients through the whole rehabilitation process by means of exercise prescriptions and educational strategies that promote exercise habits and changes in their lifestyle. The implemented design follows a new approach that intends to facilitate post M.I. patients' participation in their recovery process through the personalization of the information and offered services.

\section{METHODOLOGY}

Since cardio rehabilitation has multifactorial components the problem has been tackled following a holistic approach [5]. A multidisciplinary team formed by cardiologists, nurses, psychologists, engineers and software developers has been involved in the project in order to collaborate during all the phases of the system design. Models, specifications and the final system have been validated both with medical experts and final users. The design process has comprised four phases (research, modeling, development, and validation) that have been accomplished in an iterative way [6]. In total, five iterations have been completed in order to achieve the final design.

During the research phase a comprehensive state of the art in cardio rehabilitation has been performed, including the revision of clinical guidelines [7] and the analysis of the rehabilitation processes in three European countries (Spain, Germany and United Kingdom). This study led to the identification of the basic activities to be included in a cardiac rehabilitation program, namely medical evaluation, exercise prescription exercise, provision of education, and counseling of the patient with cardiac disease. Additionally, two protocols were selected as the reference model for the development of the system: the exercise program that currently is prescribed in the German rehabilitation clinics and the Heart Manual program of NHS Lothian in United Kingdom [8] designed to support the rehabilitation thought education and health behaviors promotion. In this sense, health belief models [9] have been taken into account in order to create a model that better promotes the exercise program, education and health behaviors. Engineers and medical experts worked together to identify the so-called "patients' desired behaviors", that represent health comportments that patients could achieve by using the system.

The modeling phase required the use of two methodologies: Agile [10] and Goal Centered Design 
[11][12]. The first one has been applied to close the model specification (e.g. exercise program and coaching strategy) while the second one has been used to define the user interaction parameters (e.g. personas, scenarios and key paths of the user interaction).

Finally, the development and validation phases have involved the implementation of five different prototypes of the system that have been subsequently assessed by experts and final users (patients and professionals).

\section{RESULTS}

The final system is a technological platform that supports a complete cardiac rehabilitation program especially focused on the physical training. The complete exercise program has been defined by health professionals and has a complete duration of six months divided in three different phases. It supports two types of exercises: endurance (walking, running or cycling) and resistance, although only the first type is monitored by the system. At the same time, the program is complemented with a behavioral change strategy based on the Heart Manual approach [8] designed to indentify patients' beliefs about their illness and help them to identify behaviors or lifestyle risks associated with these beliefs. Using different approaches, patients are empowered to change their behaviors. Additionally, the program tailors the educational material and the coaching strategy to the specific patient's individual knowledge level, self confidence, and progress.

Although the final design includes both Patient and Professional's systems, the novelty resides on the Patient's one that has been conceived to guide them during the whole rehabilitation process while empowering them to self manage their own disease (Fig.1).

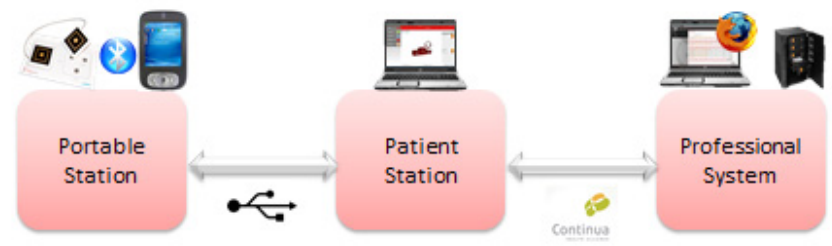

Fig. 1: Main components of the Guided Exercise System

The Professional system is used by medical professionals to prescribe a care plan basically composed by a personalized exercise program. The system also supports patients' initial assessment, provides a set of visualization tools to monitor their progress, and generate alerts in case of medical complications.

The Patient system includes two sub systems: the Portable Station and the Patient Station. The first one is a mobile device or PDA (current used model is HTC s200) complemented with a wearable sensor used to gather biomedical information during the exercise sessions (2 leads ECG, heart rate, and respiration rate). During these sessions, patients' vital signs are monitored by the sensor and transferred to the PDA via Bluetooth, while they receive, though the Portable Station, continuous guidance, instructions and personalized feedback to ensure that they train following the recommendation of the medical professionals in a safe way.

Before starting a session, the system checks the patients' medical condition using the blood pressure values and the answers of a basic questionnaire. Then, patients are asked to put on the wearable sensor and select the type of endurance exercise that they wish to perform (walking, running or cycling). The exercise itself includes three phases (warm up, core and cool down), each of them with specific target values of heart rate.

The Portable Station has been developed to promote the use of the system for exercising that is the core behavior of the rehabilitation program. During the exercise, patients are continuously supported by the system that guides them to perform the sessions in a safe and personalized way. Using the Portable Station and the wearable sensor, patients are able to follow the protocol defined by the medical experts. The system continuously checks the heart rate values and provides patients with feedback and instructions (e.g. speed up, slow down, etc.) that help them to reach and maintain their parameters in the proper range. This is done using diverse interaction modalities (e.g. graphical user interface screens, sounds and vibrations) that ensure that patients receive appropriate instructions to accomplish the planned exercise sessions.

At the end of the session patients report the level of exertion using the Borg's scale [13] and the level of enjoyment. The Portable Station evaluates the global session and provides a scoring to the exercise session. All the information gathered during the endurance training sessions is used to personalize the feedback messages about exercise progress according to the plan that medical experts personalized for each patient, and it is also transferred from the Portable Station to the Patient Station using the USB protocol.

The Patient Station is a PC based system (it runs on a tablet PC, using Java 6.1) that patients can use to check and personalize their exercise plan, analyze their performance and progress, and receive feedback and educational information. It includes a messages system that, based on personalized feedbacks, provides patients with information to help them following their training program. Through these communications, patients are able to access to their care plan, planned exercises, results and progress. The messages have been designed and scheduled in a time line according to the educational strategy defined by medical experts. The following types of messages have been defined:

- Messages to inform about the exercise plan prescribed by the doctor. These include information like type of exercise, duration, scheduling, or safe values of heart rate.

- Technical instructions to facilitate the use of the system (e.g. synchronization between the Patient Station and the Portable Station, how to wear the shirt, how to place 
the sensor, etc.).

- Feedbacks about the exercise sessions (e.g. daily results, weekly progress and global progress).

- Educational tips, a scheduled delivery of recommended educational content that has been selected by medical experts.

The Patient Station supports the exercise sessions offering patients detailed information about their progress and performance. The Results of the monitored session can be seen by individual session, on a weekly basis and as global progress. Moreover, a reward strategy has been built taking into account the global performances and the adherence to the exercise program. Special attention has been paid to the design and presentation of the information in order to motivate the patients and help them to complete their recovery process.

The system also incorporates an Educational Content Repository specifically selected by health professionals to promote some of the previously identified behaviors on patients (e.g. helping them to understand their health condition and the benefits of exercising). The majority of this content has been based on the information included in the Heart Manual created by NHS Lothian [8] and includes, among others, comprehensive information about the exercise, technical instructions for using the system, medical information about the disease and its causes, modifiable risk factors, and life style changes. This educational material is complemented by a set of Misconception Questionnaires, which are used to identify false beliefs of the patients about the exercise and the disease and try to educate them toward the right concepts. The system analyses patients' wrong answers and provides them with suggestions or tips for reading specific educational material (included in the Educational Content Repository) that can help them to increase their knowledge and eliminate the misconceptions they had. Finally, the whole system includes a virtual assistant or Avatar as an additional tool to provide patients with tips and suggestions that can help them to use the system during their recovery process. From the technical prospective, most parts of the education and motivation business logic has been developed using the following libraries: Drools (the expert system has been used to set up the feedbacks messages), JBPM (on top of the Workflow engine a User Interaction Workflow engine has been coded) and JChart (for data visualization).

Although the core of the system is the medical prescription of exercise, it is complemented with an educational and motivation strategy designed to promote certain desired behaviors. In total seven desired behaviors have been defined, all of them focused on highlighting the importance of the cardiac rehabilitation and especially the physical exercise:

- The Patient starts using the system.

- The Patient performs exercise using the system.

- The Patient understands the benefits of exercising.

- The Patient intensifies the exercise program and start exercising also without the system.

- The Patient has general knowledge about cardiovascular diseases (CVD), heart attack and risk factors.

- The Patient selects topics that are related with reduction of risk factors and learn practical tips to control them.

- The Patient continues exercising and applies the selected healthy behavior even without using the system.

For each desired behavior, the strategy defines certain activities designed to guide patients towards its achievement, as well as the specific software tools to effectively implement each task. The strategy even considers specific situations where a desired behavior cannot be achieved. The following table shows the behaviors that have been taken into account during the design of the guided exercise system, along with the associated activities and software tools (Table1).

TABLE I

DESIRED BEHAVIOR TABLE

\begin{tabular}{|c|c|c|}
\hline Desired Behavior & Summarized strategy & Software tool \\
\hline $\begin{array}{l}\text { B1: The Patient } \\
\text { starts using the } \\
\text { system }\end{array}$ & $\begin{array}{l}\text { Assist the patient } \\
\text { during first use of the } \\
\text { system. }\end{array}$ & $\begin{array}{l}\text { Introduction to the } \\
\text { system, avatar for } \\
\text { technical instructions. }\end{array}$ \\
\hline $\begin{array}{l}\text { B2: The Patient } \\
\text { performs exercise } \\
\text { using the system. }\end{array}$ & $\begin{array}{l}\text { Explain the exercise } \\
\text { program, how to } \\
\text { exercise (warm up, } \\
\text { core, cool down), } \\
\text { possible types of } \\
\text { exercise, how to report } \\
\text { the level of effort, use } \\
\text { reminders for } \\
\text { exercising. }\end{array}$ & $\begin{array}{l}\text { Assistive device to } \\
\text { monitor exercise } \\
\text { session, reminders, } \\
\text { educational content } \\
\text { about endurance } \\
\text { exercise, feedbacks on } \\
\text { exercise performance } \\
\text { and compliance. }\end{array}$ \\
\hline $\begin{array}{l}\text { B3: The Patient } \\
\text { understands the } \\
\text { benefits of } \\
\text { exercising. }\end{array}$ & $\begin{array}{l}\text { Show the progress, } \\
\text { assess the knowledge } \\
\text { about exercise, and } \\
\text { provide information } \\
\text { about benefits of } \\
\text { exercise. }\end{array}$ & $\begin{array}{l}\text { Periodic results/ } \\
\text { feedbacks of the } \\
\text { exercise, educational } \\
\text { content about } \\
\text { importance of exercise, } \\
\text { misconceptions } \\
\text { questionnaire about } \\
\text { exercise. }\end{array}$ \\
\hline $\begin{array}{l}\text { B4: The Patient } \\
\text { intensifies the } \\
\text { exercise program } \\
\text { and start } \\
\text { exercising also } \\
\text { without the } \\
\text { system. }\end{array}$ & $\begin{array}{l}\text { System introduces new } \\
\text { exercise types } \\
\text { (resistance), provides } \\
\text { information about new } \\
\text { exercises. Support } \\
\text { exercise reporting. }\end{array}$ & $\begin{array}{l}\text { Educational contents } \\
\text { about extra exercises, } \\
\text { diary for report, result } \\
\text { page, feedback on } \\
\text { progress and balance } \\
\text { with endurance } \\
\text { exercise. }\end{array}$ \\
\hline $\begin{array}{l}\text { B5: The Patient } \\
\text { has general } \\
\text { knowledge about } \\
\text { CVD, heart attack } \\
\text { and risk factors. }\end{array}$ & $\begin{array}{l}\text { Assess level of } \\
\text { knowledge, provide } \\
\text { information about } \\
\text { CVD. }\end{array}$ & $\begin{array}{l}\text { Educational content, } \\
\text { misconceptions } \\
\text { questionnaire. }\end{array}$ \\
\hline $\begin{array}{l}\text { B6: The Patient } \\
\text { selects topics that } \\
\text { are related with } \\
\text { reduction of risk } \\
\text { factors and learn } \\
\text { practical tips to } \\
\text { control them. }\end{array}$ & $\begin{array}{l}\text { The Patient selects the } \\
\text { most interesting } \\
\text { topics. }\end{array}$ & $\begin{array}{l}\text { Track patients' content } \\
\text { preferences, health } \\
\text { behaviour } \\
\text { questionnaire, and } \\
\text { educational content. }\end{array}$ \\
\hline
\end{tabular}




\begin{tabular}{lll}
\hline \hline Desired Behavior & Summarized strategy & Software tool \\
\hline $\begin{array}{l}\text { B7: The Patient } \\
\text { continues } \\
\text { exercising and }\end{array}$ & $\begin{array}{l}\text { Provide information } \\
\text { and practical tips to } \\
\text { applies the }\end{array}$ & Educational content. \\
$\begin{array}{l}\text { colinue exercising. } \\
\text { behavior even }\end{array}$ & \\
without using the & \\
system. & \\
\hline \hline
\end{tabular}

The set of tools used to promote the previously described behaviors are included both in the Patient Station and Portable Station (e.g. reminders, feedbacks, tips, questionnaires, information, and educational material).

\section{DISCUSSION AND CONCLUSION}

Each of the five developed prototypes obtained as an outcome of the followed iterative process has been validated both with experts and final users (patients and professionals) by means of interviews and questionnaires. The results of this intermediate assessment process have been very positive and the outcomes have shown high rates of acceptance for the proposed solutions.

Global acceptance for Guided Exercise system

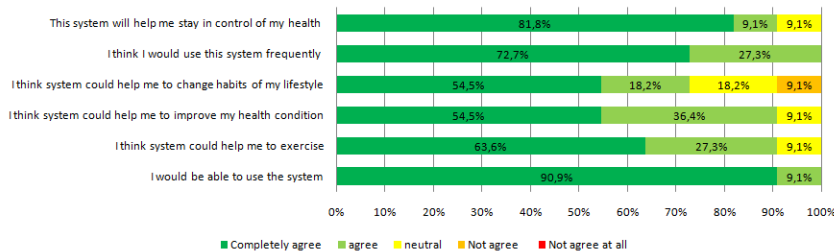

Fig. 2: Results of global acceptance questionnaire for the Guided Exercise system

The last version of the system has been evaluated with 11 patients in the Castle Hill Hospital of Kingston Upon Hull (United Kingdom). The outcomes showed that more than $80 \%$ of the participants considered the system helpful for managing their disease, thought that it could help them to exercise and were willing to use it (Fig. 2).

In the second half of 2011 this final system will be extensively evaluated in a clinical study where medical professionals intend to measure the benefits of this program compared with the standard rehabilitation process. At the same time the reliability of the defined education strategies to promote the self-behaviors will be validated using the following metrics: a) acceptability and usability of the system; b) improvement of the perception of illness, one indicator of how patients understand their medical condition; c) level of knowledge about exercise and disease; and d) exercise habits, before and after the study.

The approach that has been followed for the development of the described cardiac rehabilitation system opens a new way to develop personalized system for health care. The presented method implies more complexity on the system design, not only for the required technology (in this case, the exercise monitoring) but also on the information delivery and personalization. Its main innovation is the followed methodology, built as result of the harmonization of different knowledge domains (medical, software design, psychological, educational, user interaction, data presentation), as well as the focus on the provision of a reliable, understandable and useful support for post myocardial infarction patients during the cardio rehabilitation process.

This new approach change the focus of the care service from mere tele-monitoring platforms to behavior oriented systems in which patients are empowered. Providing healthcare systems with this kind of tools designed to promote healthy behaviors is expected to increase the patient engagement and participation in their disease management.

\section{ACKNOWLEDGMENT}

We would like to thank the whole HeartCycle Consortium for their valuable contribution to this work, especially to NHS Lothian of Edinburgh, Philips Research Eindhoven, San Carlos Hospital of Madrid, and Castle Hill Hospital of Kingston upon Hull. HeartCycle project is partially funded by the European Union.

\section{REFERENCES}

[1] Office of the Actuary in the Centers for Medicare \& Medicaid Services "National Health Expenditure Projections 2009-2019" https://www.cms.gov/NationalHealthExpendData/downloads/NHEPro jections2009to2019.pdf, last visit 20 $0^{\text {th }}$ June 2011.

[2] K. Dean, T. Hickey \& B.E. Holstein, "Self-care and health in old age", Billing \& sons Limited, Worcester, 1986, pp. 37-52.

[3] Thomas Goetz, "The Decision Tree: Taking Control of Your Health in the New Era of Personalized Medicine", Rodale Books, February 2010.

[4] European Commission. Information Society Technologies Program. HeartCycle project. Compliance and effectiveness in HF and CHD closed-loop management. FP7 - 216695. http://heartcycle.eu/, last visit 20th June 2011.

[5] American Association of cardiovascular and pulmonary rehabilitation, "Core Competencies for Cardiac Rehabilitation/Secondary Prevention Professionals: 2010 Update", August 2010

[6] IABG Information Technology, "V-Model, Lifecycle Process Model", 1995; http://www.v-modell.iabg.de/kurzb/vm/k_vm_e.doc, last visit 20th June 2011.

[7] American College of Sport Medicine, "Guidelines for Exercise Testing and Prescription", Lippincott Williams \& Wilkins 2009.

[8] Heart Manual (Coronary Artery Disease Edition) @ Lothian Health Board 2008 -2nd impression. Published by Lothian Health Board, Astley Ainslie Hospital, Grange Loan, Edinburgh EH9 2HL. Edited by Dr Jo Hargreaves.

[9] Conner, M. \& Norman, P. "Predicting Health Behavior. Search and Practice with Social Cognition Models". Open University Press: Ballmore: Buckingham, 1996.

[10] Dean Leffingwell "Agile Software Requirements: Lean Requirements Practices for Teams, Programs, and the Enterprise ", Addison-Wesley Professional, January 2011.

[11] ISO 13407. Human-centred design processes for interactive systems, 1999; ISO, Geneva, Switzerland.

[12] Cooper Alan, Reimann Robert, Cronin David. "About Face 3: The Essentials of Interaction Design", 2007; Wiley3, Indianapolis.

[13] Borg, G., "Borg's Perceived Exertion and Pain Scales", 1998; Champaign, IL: Human Kinetics. 